•编者按・

\title{
亚洲植物多样性数字化计划
}

\author{
马克平 ${ }^{*}$
}

(中国科学院植物研究所植被与环境变化国家重点实验室, 北京 100093)

\section{Mapping Asia Plants: a cyberinfrastructure for plant diversity in Asia}

\author{
Keping $\mathrm{Ma}^{*}$ \\ State Key Laboratory of Vegetation and Environmental Change, Institute of Botany, Chinese Academy of Sciences, Bei- \\ jing 100093
}

近年来, 生物多样性信息学快速发展, 全球和 区域水平的生物多样性数据库不断建立和完善。用 关键词“biodiversity”和“database”在Web of Science 检索可知, 2016年发表的生物多样性数据库相关的 论文数量是 20 年前即 1997 年的 22 倍, 是 10 年前即 2007年的2倍(附录1)。全球生物多样性信息网络 (GBIF) 等全球大型数据库, 以及若干国家水平的数 据库，如澳大利亚生物多样性信息系统(Atlas of Living Australia, ALA)和美国标本数字化平台 (iDigBio)等, 都可以提供数千万条物种分布信息, GBIF可以提供 7 亿多条物种分布信息(植物信息只 占约 $1 / 4)$ 。海量的物种分布数据为生物多样性科学 研究提供了有力支撑, 大大促进了生物多样性大尺 度格局、生物多样性保护规划、生物多样性对全球 变化的响应、外来种入侵态势预测等方面的研究。 从全球生物多样性在线数据资源分布看, 亚洲整体 上属于数据贫乏的区域, GBIF的植物分布数据亚洲 只占 $4 \%$ 。截至目前, 亚洲还没有区域尺度的生物多 样性数据库。尽管中国大陆和台湾、印度、日本、 韩国等在生物多样性数据库建设方面有比较好的 基础, 但大多数亚洲国家尚没有比较完整的生物多 样性数据库, 严重影响了亚洲区域尺度的生物多样 性研究和保护。鉴于此, 中国科学院生物多样性委 员会主导的亚洲生物多样性保护与信息网络 (ABCDNet, www.abcdn.org/)在2015年11月的年度工 作会议上通过了中方提出的亚洲植物多样性数字 化计划(Mapping Asia Plants, MAP), 经费上得到了 中国科学院国际合作局和东南亚生物多样性研究
中心的大力支持。

与 MAP类似的项目为数不多。全球尺度上有 Map of Life (MOL, www.mol.org/), 设置4个模块, 分别是物种分布图(map species)、按照地点查物种 (species by location)、变化趋势指标(indicator)和手 机应用(mobile app)模块。截至2017年2月6日, 该网 站上已有 279 个数据集 5.56 亿条记录, 涉及 84.5 万个 物种。目前提供信息服务的类群主要有兽类、鸟类、 龟鳖类(不包括海洋种)、两栖类、蝶类、天蛾类、 松柏类和仙人掌类, 主要是动物方面的, 植物方面 信息比较少。MOL旨在搜集和整合全球物种分布及 其动态变化的数据和知识, 为生物多样性教育、保 护、研究和科学决策服务。其理念是把生物多样性 画在图上(putting biodiversity on the map)。近年来, 通过普及手机客户端应用有效地推动了公众科学 在发现、鉴定和记录物种方面的贡献。在数据挖掘 上, 将物种分布模型与不断更新的遥感数据结合展 示了良好的发展前景。在区域尺度上，植物学信息 与生态网络 (Botanical Information and Ecology Network, BIEN, http://bien.nceas.ucsb.edu/bien/)提供 了很好的参考案例。BIEN的目标在于: (1)建立针对 跨尺度综合分析的科学家、数据管理者和信息技术 专家的合作网络; (2)搜集植物学信息，包括物种、 多度和性状数据, 主要是美洲的植物; (3)开发在线 信息技术平台以推动植物信息的发现、研究和管 理。BIEN2.0版的数据集包括1,217.1万个观测记录、 934.5万份数字化标本、33万个植被样方、14万个性 状数据, 涉及20.1万个物种。目前, BIEN的数据只对

\footnotetext{
* 通讯作者 Author for correspondence. E-mail: kpma@ibcas.ac.cn
} 
网络成员开放, 计划将来对社会开放。

MAP旨在建立亚洲植物学信息的大数据在线 平台, 为亚洲植物多样性保护与研究提供综合性基 础信息和跨学科数据挖掘环境。第一阶段 (2016-2020年)的重点在于建立亚洲高等植物物种 名录与分布数据库, 并开发数据管理和挖掘的在线 平台, 逐步整合植物性状数据、分子-组学数据、古 植物学数据、环境数据和遥感数据。植物物种名录 与分布数据库是本计划的核心, 数据来源主要有: (1)植物志书、植物名录和相关论文; (2)植物分布图; (3)植物标本, 包括鉴定可靠的植物照片; (4)植被调 查样方; (5)植物考察资料; (6)植物观测资料, 包括 植物爱好者发布的数据; (7)数字化的在线数据集。 除建设综合性数据平台来服务于植物生态学、进化 植物学、植物地理学、保护生物学和全球变化生物 学研究之外, 计划形成下列产品或者服务: (1)植物 物种分布图; (2)植物多样性分布图; (3)国家/区域植 物物种名录; (4)植物学名校订和物种分布模型等数 据清理与挖掘工具包和在线综合分析平台。

ABCDNet工作组和年度工作会议负责审议和 指导MAP工作组的工作。MAP工作组由组长和7名 成员组成, 分别负责东南亚、南亚、西亚、中亚、 北亚(俄罗斯亚洲部分)和东北亚的组织协调, 以及 在线平台开发(附录2)。每个成员负责搜集相关地区 的植物名录与分布信息, 形成初步材料, 然后邀请 相关国家的植物分类学家审订完善。目前, 除西亚 外, 各个地区的进展都比较好。2016年11月在北京 召开了MAP东南亚植物多样性信息研讨会 (www.abcdn.org/news/3126), 来自8个国家的植物分 类学专家参加了会议; 今年计划召开针对南亚和亚 洲北部(中亚、北亚和东北亚)地区的专题研讨会。

在基础相对薄弱的亚洲, 开展区域尺度植物多 样性数据库建设充满了挑战。很多数据集都未公开
发表或发布, 特别是植物分布数据很多是当地语 言, 而且难以搜集到; 大多数国家的植物学研究都 是境外专家为主完成的, 有时甚至连找到相关的分 类学家都很困难, 索取资料就更难; 研究水平参差 不齐, 再加上持续了上百年时间, 物种分类学概念 的变化和多源异构数据汇总后的清理与整合难度 很大; 与发达国家相比, 亚洲的数据共享精神相对 薄弱，增加了信息搜集的难度和使用中知识产权问 题的复杂性; 目前中国政府的研究经费管理制度不 利于跨境项目, 难以申请到大额度经费, 而且经费 境外使用难度较大; 现行的科研评价体系不利于开 展这样的基础性工作, 花费时间长、短期内很难发 表高水平的文章, 需要对MAP的未来充满信心的成 员加盟, 增加了团队建设的难度。

尽管存在诸多挑战，但中国牵头启动MAP的时 机已经成熟。中国在植物分类和分布方面的科学积 累是亚洲最好的, 《中国植物志》和Flora of China 等志书的出版, 以及 《中国生物物种名录》植物部 分的不断更新已奠定了坚实的基础; 在生物多样性 信息学方面也是亚洲最有优势的, 如数字化的植物 标本达到九百多万份(www.nsii.org.cn/), 是GBIF中 所有亚洲植物标本总和的 1.5 倍; 基于大型植物分 布数据集发表的植物地理学、保护生物学、入侵生 物学和全球变化生物学等方面的高水平学术论文 也是亚洲国家最多的; 在科学优势的基础上, 通过 多年国际交流建立起来的亚洲植物学同行的合作 网络, 特别是2013年建立的ABCDNet为MAP项目 的开展创造了条件; 中国科学院陆续建立的海外研 究中心为MAP项目的开展提供了重要支持; 中国 政府以“走出去”为标志的新一轮开放战略, 是MAP 等区域性项目得以施行的政治基础。因此, 有理由 相信, 亚洲植物多样性数字化计划定会在各个方面 的合作与支持下健康稳步发展。

\section{附录 Supplementary Material}

附录1 二十年来生物多样性数据库相关论文数量变化趋势

Appendix 1 Trends of number of papers on biodiversity database published in the past 20 years (http://www.biodiversity-science.net/fileup/PDF/2017030-1.pdf)

附录2 亚洲植物多样性数字化计划工作组

Appendix 2 Working group for Mapping Asia Plants

(http://www.biodiversity-science.net/fileup/PDF/2017030-2.pdf) 


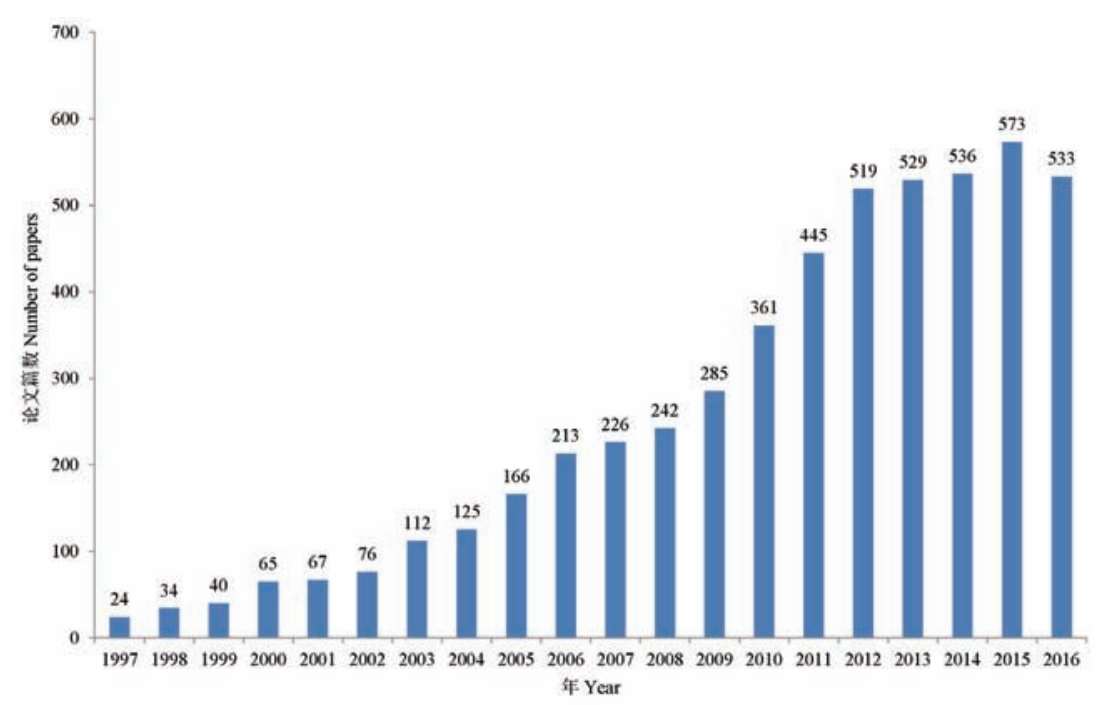

附录1 二十年来生物多样性数据库相关论文数量变化趋势。用关键词“biodiversity”和“database”在Web of Science检索(检索时间: 2017年2月5日)

Appendix 1 Trends of number of papers on biodiversity database published in the past 20 years. 
马克平. 亚洲植物多样性数字化计划. 生物多样性, 2017, 25 (1): 1-2.

附录2 亚洲植物多样性数字化计划工作组

Appendix 2 Working group for Mapping Asia Plants

组长: 罗茂芳(maofang@ibcas.ac.cn)

成员：李文军(liwenjunao@ 126.com), 负责中亚

刘 博(boliu@muc.edu.cn), 负责东南亚

林秦文(linqinwen83@163.com), 负责南亚

王洪峰(wanghongfeng90@163.com), 负责东北亚

徐学红(strawberry@ibcas.ac.cn), 负责西南亚

薛建华(xuejianhua@ibcas.ac.cn), 负责北亚

许哲平(xuzp@ibcas.ac.cn), 负责信息技术 